\title{
The effectiveness of an enhanced recovery after surgery protocol in head and neck cancer surgery with free-flap reconstruction
}

\author{
Ho-Ryun Won', Jun-Young An², Jung Jun Lee², Dong Young Kim², Jeon Yeob Jang², Chul-Ho Kim², \\ Yoo Seob Shin ${ }^{2}$ \\ ${ }^{1}$ Department of Otolaryngology-Head and Neck Surgery, Chungnam National University Hospital, Daejeon, Korea \\ ${ }^{2}$ Department of Otolaryngology, Ajou University School of Medicine, Suwon, Korea
}

\begin{abstract}
Purpose: An enhanced recovery after surgery (ERAS) protocol incorporates up-to-date perioperative care principles; the primary aim in using an ERAS protocol is to reduce issues that delay the recovery and cause the complications. The aim of this study was to compare outcomes associated with head and neck cancer surgery with free-flap reconstruction before and after implementation of an ERAS protocol.

Methods: Outcomes were analyzed by dividing patients into 2 groups: 29 patients in the non-ERAS group and 60 patients in the ERAS group. The ERAS group performed a prospective observational cohort study of patients who underwent a head and neck cancer surgery with free-flap reconstruction in Ajou University Hospital from August 2015 to December 2017. The non-ERAS group retrospectively reviewed the medical records of patients who had undergone the same surgery from August 2012 to July 2015.

Results: Demographics, comorbidities, hospital length of stay (LOS), postoperative complications, starting time of rehabilitation, and postoperative periods before radiotherapy for the non-ERAS and ERAS groups were compared. Hospital LOS was significantly lower for patients whose care followed the ERAS protocol than for patients in the non-ERAS group (30.87 \pm 20.72 days vs. $59.66 \pm 40.43$ days, $P<0.0001)$.

Conclusion: In this study, hospital LOS was reduced through fast recovery after the implementation of the ERAS protocol. Therefore, the ERAS protocol appeared feasible and safe in head and neck cancer surgery with free-flap reconstruction.

[Ann Surg Treat Res 2019;97(5):239-244]
\end{abstract}

Key Words: Adjuvant radiotherapy, Free tissue flaps, Head and neck neoplasms, Perioperative care, Postoperative care

\section{INTRODUCTION}

The postoperative recovery process has recently been considered as important as the surgical procedure itself. Therefore, multimodal and multidisciplinary team approaches are needed to reduce recovery period and surgical complications [1-3]. The concept of an enhanced recovery after surgery (ERAS) program was first proposed as a fast-track multimodal program for inpatients with colorectal cancer and has since been applied to several types of surgical procedure [4].

Head and neck cancer surgery with free-flap reconstruction which requires functional reconstruction after extensive resection, necessitates considerable time and effort in the surgical procedure, and is followed by a long recovery period. Therefore, multidisciplinary perioperative care is needed to ensure rapid recovery and rehabilitation $[1,2,5]$. The ERAS
Received June 27, 2019, Revised September 4, 2019 ,

Accepted October 8, 2019

Corresponding Author: Yoo Seob Shin

Department of Otolaryngology, Ajou University School of Medicine, 164

Worldcup-ro, Yeongtong-gu, Suwon 16499, Korea

Tel: +82-31-219-5262, Fax: +82-31-219-5264

E-mail: ysshinmd@ajou.ac.kr

ORCID: https://orcid.org/0000-0002-2007-1100
Copyright (c) 2019, the Korean Surgical Society

(c) Annals of Surgical Treatment and Research is an Open Access Journal. All articles are distributed under the terms of the Creative Commons Attribution NonCommercial License (http://creativecommons.org/licenses/by-nc/4.0/) which permits unrestricted non-commercial use, distribution, and reproduction in any medium, provided the original work is properly cited. 
Society has presented guidelines for various surgical procedures since it was established in 2010; a guideline for head and neck cancer surgery with free-flap reconstruction was proposed in 2016 [2]. The specific perioperative care elements of the ERAS protocol proposed for head and neck cancer surgery with free-flap reconstruction are based on the items in the ERAS protocols for other surgical procedures [2]. However, effectiveness of the ERAS protocol for head and neck cancer surgery with free-flap reconstruction requires verification through the collection and evaluation of clinical data following implementation of the protocol.

Therefore, the aims of this study were to establish the level of progress in the implementation of the basic ERAS protocol principles for various surgical procedures to head and neck cancer surgery and to evaluate the outcomes following ERAS protocol utilization in the head and neck cancer surgery with free-flap reconstruction.

\section{METHODS}

\section{Patients groups}

We performed a prospective observational cohort study in a total of 60 patients who underwent head and neck cancer surgery with free-flap reconstruction in the head and neck surgery department from August 2015 through December 2017 at Ajou University Hospital and whose care followed the ERAS protocol. A total of 29 patients who underwent reconstructive surgery before July 2015 and whose care followed without the ERAS protocol were selected as non-ERAS group and compared to ERAS group. The non-ERAS group collected and analyzed data through a retrospective chart review.

\section{Principles of the ERAS protocol in Ajou University Hospital}

Before implementation of the ERAS approach, perioperative care was directed by the individual surgeon and did not follow a specific protocol. Beginning in August 2015, a protocol was established according to the basic ERAS principles and applied to perioperative care. The main contents of the Ajou Hospitaldeveloped ERAS protocol for head and neck cancer surgery with free-flap reconstruction patients were as follows. Patients were informed regarding the surgical procedure and precautions before surgery, and the operation proceeded with voluntary consent. Prophylactic intravenous antibiotics were administered 1 to 2 hours before surgery and preanesthetic medication was administered before anesthesia. Patients were routinely admitted to the intensive care unit (ICU) on the operation day, and ventilator weaning took place immediately if there were no cardiopulmonary difficulties. If there were no specific problems or complications on postoperative day (POD) 1, the patient was transferred to the general ward. Routine anticoagulation was performed with low-molecular weight heparin or alprostadil for 2 days through a central intravenous line. The urinary catheter and central intravenous line were removed on POD 1. Fluid replacement was managed in a goal-directed manner while avoiding excessive over- or underhydration. A standard polymeric enteral nutrition formula was administered starting on POD 1, and oral feeding training was started on POD 5 with sips of water while maintaining enteral nutrition. A cold liquid diet was started on POD 7, and enteral nutrition was discontinued when sufficient nutrition was satisfied by oral feeding. Immediately after the operation, analgesics were administered through the intravenous line to manage patient pain; paracetamol and available nonsteroidal anti-inflammatory drugs were used in combination. Opioid analgesics were also used at the physician's discretion. Analgesics were appropriately controlled during the hospitalization period according to the severity of patient's pain. A single postoperative antibiotic was administered through POD 7. Postoperative mobilization was enacted as soon as possible. Normally, the patient maintained a sitting position until wheelchair ambulation on POD 5 , usually after maintaining absolute bedrest through POD 2 . Patients began ward ambulation on POD 7. Flap monitoring was performed at 2-hour intervals for 2 days after surgery, and every 12 hours thereafter.

\section{Evaluation index}

The medical information of patients who underwent head and neck cancer surgery with free-flap reconstruction was analyzed to determine an evaluation index. This study was approved by the Institutional Review Board of Ajou University Hospital (AJIRB-18-050). Patient demographics, including age, sex, comorbidities, and body mass index (BMI) were collected, and preoperative American Society of Anesthesiologists (ASA) physical status classification, were evaluated. The World Health Organization score and Karnofsky score (a form of performance score) were also assessed. In addition, each patient's head and neck cancer subsite, cancer staging (American Joint Committee on Cancer, 7th edition), total surgery duration and free-flap reconstructive surgery duration were collected.

Outcomes for the ERAS and non-ERAS groups were evaluated on the following factors. The ICU length of stay (LOS) and the hospital LOS were assessed. The starting times of adjuvant radiotherapy, oral feeding, and normal ambulation were measured. In addition, postoperative complications, such as surgical wound complications, pneumonia, delirium, and expiration, were analyzed.

\section{Statistical analysis}

Patients demographics are expressed as number and frequency (\%), and continuous variables such as age, BMI, and outcome index are reported as means \pm standard deviation. 
The chi-square test and Fisher extract test were used for comparison of categorical variables, while the Student's t-test was used for comparison of continuous variables. Multiple regression analysis was performed. Statistical significance was set at a $\mathrm{P}<0.05$. Data were collated and analyzed with IBM SPSS Statistics ver. 20.0 (IBM Co., Armonk, NY, USA).

\section{RESULTS}

\section{Demographics and patient characteristics}

Table 1 presents a comparison of patient characteristics between the ERAS and non-ERAS groups. Age, sex, preoperative status, and performance score were not significantly different between the 2 groups. Comorbidities, subsites of head and neck cancer, and cancer stage were also not significantly different. However, there was a significant difference in BMI between the ERAS group (23.09 \pm 3.17$)$ and the non-ERAS group (22.58 \pm

Table 1. Patient characteristics

\begin{tabular}{|c|c|c|c|c|}
\hline Characteristic & All patients $(\mathrm{n}=89)$ & Non-ERAS $(\mathrm{n}=29)$ & ERAS $(n=60)$ & P-value \\
\hline Age $(y r)$ & $59.27 \pm 11.57$ & $59.24 \pm 12.37$ & $59.28 \pm 11.28$ & 0.987 \\
\hline Male sex & $66(74)$ & $25(86)$ & $41(68)$ & 0.071 \\
\hline Body mass index $\left(\mathrm{kg} / \mathrm{m}^{2}\right)$ & $22.58 \pm 3.32$ & $21.52 \pm 3.44$ & $23.09 \pm 3.17$ & $0.036^{*}$ \\
\hline ASA PS classification $\geq$ III & $12(13)$ & $1(3)$ & $11(18)$ & 0.094 \\
\hline WHO score $\geq$ II & $7(8)$ & $0(0)$ & $7(12)$ & 0.091 \\
\hline Karnofsky score $\leq 60$ & $5(6)$ & $0(0)$ & $5(8)$ & 0.169 \\
\hline Hypertension & $36(40)$ & $11(38)$ & $25(42)$ & 0.820 \\
\hline Diabetes & $13(15)$ & $4(14)$ & $9(0.15)$ & $>0.999$ \\
\hline Ischemic heart disease & $6(7)$ & $1(3)$ & $5(8)$ & 0.659 \\
\hline \multicolumn{5}{|l|}{ Diagnosis } \\
\hline Oral cavity cancer & $41(46)$ & $16(55)$ & $25(42)$ & 0.263 \\
\hline Oropharynx cancer & $16(18)$ & $6(21)$ & $10(17)$ & 0.769 \\
\hline Hypopharynx cancer & $7(8)$ & $1(3)$ & $6(10)$ & 0.420 \\
\hline Supraglottic cancer & $7(8)$ & $1(3)$ & $6(10)$ & 0.420 \\
\hline Others & $18(20)$ & $5(17)$ & $13(22)$ & 0.781 \\
\hline \multicolumn{5}{|l|}{ Stage } \\
\hline I & $1(2)$ & $1(3)$ & $0(0)$ & 0.326 \\
\hline II & $9(10)$ & $3(10)$ & $6(10)$ & $>0.999$ \\
\hline III & $10(11)$ & $2(7)$ & $8(13)$ & 0.489 \\
\hline IV & $66(74)$ & $22(76)$ & $44(73)$ & 0.789 \\
\hline
\end{tabular}

Values are presented as mean \pm standard deviation or number $(\%)$.

ERAS, enhanced recovery after surgery; ASA PS, American Society of Anesthesiologists physical status; WHO, World Health Organization.

$* \mathrm{P}<0.05$, significant difference.

Table 2. Comparison of control to ERAS group

\begin{tabular}{lccc}
\hline \multicolumn{1}{c}{ Variable } & Non-ERAS $(\mathrm{n}=29)$ & ERAS $(\mathrm{n}=60)$ & P-value \\
\hline ICU LOS (day) & $9.48 \pm 7.13$ & $1.20 \pm 0.92$ & $<0.001^{*}$ \\
Hospital LOS (day) & $59.66 \pm 40.43$ & $30.87 \pm 20.73$ & $<0.001^{*}$ \\
Ambulation start (day) & $23.78 \pm 20.25$ & $6.65 \pm 3.27$ & $<0.001^{*}$ \\
Oral feeding start (day) & $23.4 \pm 10.29$ & $15.72 \pm 19.05$ & $<0.001^{*}$ \\
Adjuvant radiation start (day) & $54.06 \pm 23.18$ & $37.93 \pm 8.65$ & $<0.001^{*}$ \\
Pneumonia & $7(24)$ & $15(25)$ & $>0.999$ \\
Delirium & $12(41)$ & $16(27)$ & 0.223 \\
Surgical wound complication & $4(14)$ & $6(10)$ & 0.722 \\
Expired & $2(7)$ & $0(0)$ & 0.104 \\
\hline
\end{tabular}

Values are presented as mean \pm standard deviation or number $(\%)$.

ERAS, enhances recovery after surgery; ICU, intensive care unit; LOS, length of stay.

$* \mathrm{P}<0.05$, significant difference. 


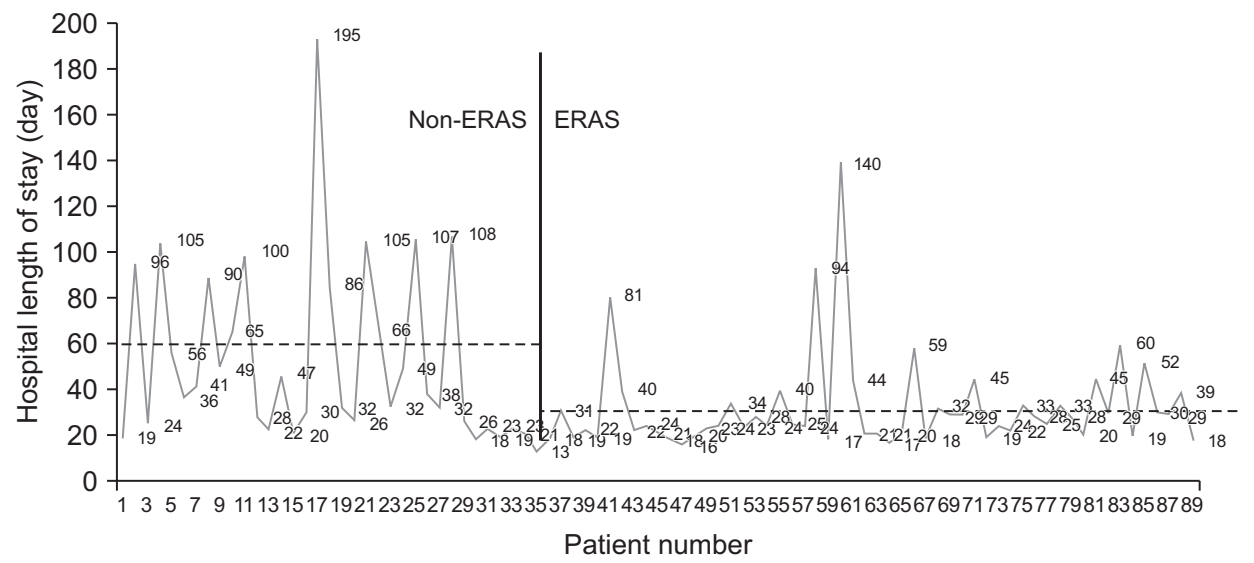

Fig. 1. Hospital LOS of all patients. The mean hospital LOS of 27 patients in the non-ERAS protocol group was $59.66 \pm 40.43$ days, and the mean hospital LOS of 60 patients in the ERAS protocol group was $30.87 \pm 20.73$ days $(P<0.001)$. ERAS, enhanced recovery after surgery; LOS, length of stay.

Table 3. Multiple regression analysis

\begin{tabular}{clcccc}
\hline Dependent variable & Independent variable & $\mathrm{B}$ & $\beta$ & $\mathrm{t}$-value & P-value \\
\hline Hospital LOS & ICU LOS & 1.076 & 0.223 & 2.528 & $0.013^{*}$ \\
& Oral feeding start & 0.845 & 0.569 & 8.151 & $<0.001^{*}$ \\
& Ambulation start & 0.558 & 0.269 & 3.103 & $0.003^{*}$ \\
& ASA PS classification & 12.295 & 0.162 & 2.407 & $0.018^{*}$ \\
& & & &
\end{tabular}

ICU, intensive care unit; LOS, length of stay; ASA PS, American Society of Anesthesiologists physical status; B, nonstandardized beta coefficient; $\beta$, standardized beta coefficient; $R^{2}$, coefficient of determination; $R_{\text {adj; }}^{2}$ adjusted coefficient of determination.

$* \mathrm{P}<0.05$, significant difference.

3.32) $(P=0.036)$.

\section{Clinical outcomes}

The mean length of ICU LOS was $1.20 \pm 0.92$ days for patients whose care followed the ERAS protocol, which was significantly lower than the $9.48 \pm 7.13$ days for the non-ERAS group $(\mathrm{P}<0.0001)$ (Table 2). A significant difference in mean hospital LOS was also observed between the ERAS group $(30.87 \pm 20.73)$ and the non-ERAS group $(59.66 \pm 40.43)(P<$ 0.001 ) (Fig. 1). The starting times of adjuvant radiotherapy, oral feeding, and normal ambulation were significantly reduced in the ERAS group $(\mathrm{P}<0.001)$. Complications related to surgery, including pneumonia, delirium, expiration, and surgical wound complications, were not correlated with application of the ERAS protocol (Table 2).

Multiple regression analysis was performed on all indexes, with hospital LOS as a dependent variable. The starting time of oral feeding $(\mathrm{P}<0.001)$ and normal ambulation $(\mathrm{P}=0.003)$ showed significant correlation with hospital LOS, and ICU LOS $(\mathrm{P}=0.013)$ and preoperative ASA physical status classification $(\mathrm{P}=0.018)$ were also significantly correlated with hospital LOS. The coefficient of determination $\left(R^{2}\right)$ of hospital LOS for these 4 indexes was 0.65 , demonstrating reliability of about $65 \%$ (Table 3). On the other hand, total surgery duration and free-flap reconstructive surgery duration were not closely related to the extended hospital LOS.

\section{DISCUSSION}

The concept of ERAS was described by Kehlet [4,6] in the late 1990s in a series of papers on fast-track multimodal programs in colorectal surgery. The ERAS study group was organized in 2001, and the importance of its mission attracted attention. Since the ERAS Society was established in 2010, it has addressed many surgical fields, and an ERAS protocol for head and neck cancer has been proposed [7].

Currently, ERAS is not a simple, fast-track program. The basic principles of ERAS call for a multidisciplinary and collaborative approach to reduce the stress response to surgery and accelerate recovery. According to a meta-analysis of the literature, hospital LOS decreased significantly after ERAS protocol implementation, and postoperative complications decreased by $30 \%$ [8]. The similar results were shown for liver resections and for pancreatic, major urologic, gynecologic, orthopedic, and emergency surgery [7,9-11]. However, there is little available literature on the effectiveness of the ERAS protocol in head and neck cancer surgery with free-flap reconstruction. Since August 2015, our institution has employed an ERAS protocol that is based on other surgical protocols. This study performed a prospective observational study and analyzed the effects of the 
ERAS protocol.

The main feature of the ERAS protocol applied by our institution is to reduce the ICU LOS as much as possible and to transfer to the general ward faster than before implementation of the ERAS protocol. In addition, through cooperation with various departments, the protocol was set up for the purpose of rapid rehabilitation. As a result, there was a significant decrease in hospital LOS after implementation of the ERAS protocol, which was confirmed to be related to early oral feeding and ambulation. It was also found that early transfer to the general ward resulted in a reduction in hospital LOS. However, the frequency of complications in this study was not related to utilization of the ERAS protocol. Although there was a little difference in BMI between the ERAS protocol group and the control group, BMI was not significantly correlated with decreased hospital LOS in the multiple regression analysis, statistically. In addition, because of the different patient collection periods between the ERAS and non-ERAS groups, the surgeon's performance may affect the results of the study. Therefore, multivariate analysis was performed on total surgery time and reconstructive surgery time that indirectly reflected the performance of the operator. As a result, the total time of whole surgery (correlation coefficient $=0.344$ ) and the free-flap reconstructive surgery time (correlation coefficient $=0.432$ ) were not closely related to the hospital LOS. However, future studies should be conducted to limit this bias.

One of the features of head and neck cancer is the need for adjuvant radiotherapy depending on the results of postoperative histologic examination [12]. Therefore, delayed adjuvant radiotherapy caused by delays in postoperative recovery has a detrimental effect on prognosis. Implementation of the ERAS protocol significantly reduced the time to start of adjuvant radiotherapy, and this is expected to have a beneficial effect on prognosis. Therefore, prospective long-term studies should be conducted to evaluate the effects of the ERAS protocol on longterm prognosis.

The reason the hospital LOS is longer than that seen in other countries after implementation of the ERAS protocol is thought be the somewhat inexpensive hospitalization costs associated with the national insurance policy in Republic of Korea. However, hospital LOS was significantly reduced from $59.66 \pm 40.43$ days to $30.87 \pm 20.73$ days following implementation of the ERAS protocol. Although a cost analysis was not included in this study, it is considered that utilization of the ERAS protocol is associated with a decrease in hospital costs. It is recommended that future studies include a cost analysis.

In conclusion, the ERAS protocol is a multidisciplinary program of patient care during the surgical period to increase safety and comfort while reducing hospitalization time. Utilization of the ERAS protocol in head and neck cancer surgery with free-flap reconstruction reduced the hospital LOS and reduced the time to start of adjuvant radiotherapy. In conclusion, the ERAS protocol should be considered for implementation in head and neck cancer surgery that includes reconstruction.

\section{CONFLICTS OF INTEREST}

No potential conflict of interest relevant to this article was reported.

\section{ACKNOWLEDGEMENTS}

This work was supported by the 2016 New Faculty Research Fund of Ajou University School of Medicine.

\section{REFERENCES}

1. Coyle MJ, Main B, Hughes C, Craven R, Alexander R, Porter G, et al. Enhanced recovery after surgery (ERAS) for head and neck oncology patients. Clin Otolaryngol 2016;41:118-26.

2. Dort JC, Farwell DG, Findlay M, Huber GF, Kerr P, Shea-Budgell MA, et al. Optimal perioperative care in major head and neck cancer surgery with free flap reconstruction: a consensus review and recommendations from the enhanced recovery after surgery society. JAMA Otolaryngol Head
Neck Surg 2017;143:292-303.

3. Kwag SJ, Kim JG, Kang WK, Lee JK, Oh ST. The nutritional risk is a independent factor for postoperative morbidity in surgery for colorectal cancer. Ann Surg Treat Res 2014;86:206-11.

4. Kehlet H. Multimodal approach to control postoperative pathophysiology and rehabilitation. Br J Anaesth 1997;78:606-17.

5. Bianchini C, Pelucchi S, Pastore A, Feo CV, Ciorba A. Enhanced recovery after surgery (ERAS) strategies: possible advantages also for head and neck surgery patients? Eur Arch Otorhinolaryngol 2014:271:43943.

6. Kehlet H. Organizing postoperative accelerated recovery programs. Reg Anesth 1996;21(6 Suppl):149-51.

7. Ljungqvist O, Scott M, Fearon KC. Enhanced recovery after surgery: a review. JAMA Surg 2017;152:292-8.

8. Nicholson A, Lowe MC, Parker J, Lewis SR, Alderson P. Smith AF. Systematic review and meta-analysis of enhanced recovery 
programmes in surgical patients. Br J Surg 2014;101:172-88.

9. Xu W, Daneshmand S, Bazargani ST, Cai J, Miranda G, Schuckman AK, et al. Postoperative pain management after radical cystectomy: comparing traditional versus enhanced recovery protocol pathway. J Urol 2015;194:1209-13.
10. Jeong O, Ryu SY, Park YK. Postoperative functional recovery after gastrectomy in patients undergoing enhanced recovery after surgery: a prospective assessment using standard discharge criteria. Medicine (Baltimore) 2016;95:e3140.

11. Sjetne IS, Krogstad U, Odegard S, Engh ME. Improving quality by introducing en- hanced recovery after surgery in a gynaecological department: consequences for ward nursing practice. Qual Saf Health Care 2009;18:236-40.

12. Bernier J, Cooper JS. Chemoradiation after surgery for high-risk head and neck cancer patients: how strong is the evidence? Oncologist 2005;10:215-24. 